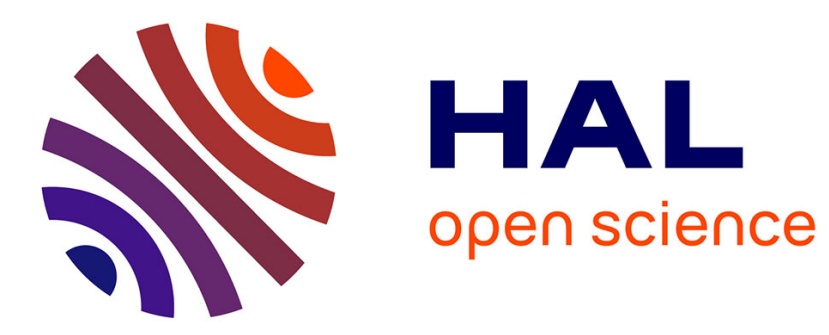

\title{
Polynomial mode approximation for longitudinal wave dispersion in circular rods
}

Denis Brizard, Eric Jacquelin, Sylvie Ronel

\section{To cite this version:}

Denis Brizard, Eric Jacquelin, Sylvie Ronel. Polynomial mode approximation for longitudinal wave dispersion in circular rods. Journal of Sound and Vibration, 2019, 439, pp. 388-397. 10.1016/j.jsv.2018.09.062 . hal-01915344

\section{HAL Id: hal-01915344 \\ https://hal.science/hal-01915344}

Submitted on 7 Nov 2018

HAL is a multi-disciplinary open access archive for the deposit and dissemination of scientific research documents, whether they are published or not. The documents may come from teaching and research institutions in France or abroad, or from public or private research centers.
L'archive ouverte pluridisciplinaire HAL, est destinée au dépôt et à la diffusion de documents scientifiques de niveau recherche, publiés ou non, émanant des établissements d'enseignement et de recherche français ou étrangers, des laboratoires publics ou privés. 


\title{
Polynomial mode approximation for longitudinal wave dispersion in circular rods
}

\author{
D. Brizard ${ }^{1, *}$, E. Jacquelin ${ }^{1}$, S. Ronel ${ }^{1}$ \\ ${ }^{a}$ Univ Lyon, Université Claude Bernard Lyon 1, IFSTTAR, LBMC UMR_T9406, F69622, Lyon, France
}

Keywords: wave propagation, longitudinal mode, dispersion equation, Pochhammer equation, polynomial approximation

\section{Introduction}

The dispersive nature of the longitudinal wave propagation in rods has been studied extensively theoretically as well as experimentally by many authors (see e.g. 1] or 2]). Split Hopkinson Pressure Bars (SHPB) is employed to dynamically characterize materials in the intermediate range of strain rate and the post-processing of the SHPB tests requires the knowledge of the dispersion in the bars [3] [4] [5]. Simplified models of longitudinal wave propagation have been proposed by Love [6] and later by Mindlin et al. [7].

In his paper published in 2006, Anderson [8] developed a rod theory for the propagation of longitudinal waves in slender rods of circular cross section and it is an improvement from the previous works in 7]. In the approximation of the longitudinal and radial displacements he uses only the first two modes of the given basis for each displacement, this leads to the four-mode equation. The four-mode equation is a polynomial whose root is the wave longitudinal velocity. This equation is therefore easier to solve than the Pochhammer equation [1] because the latter is transcendental.

However we noticed an error in one of the coefficients of the four-mode equation [8], and this error is not a mere typo. This error was also repeated in [9]. The aim of this paper is threefold. First we want to give the correct four-mode equation and the associated dispersion curve. Secondly, we wanted to examine the convergence of the approximation at higher orders then given by Anderson. Thirdly, this is the occasion to give dimensionless equations.

The paper is organised as follows: Section 2 details how to obtain the dimensionless approximation for longitudinal waves. Section 3 recalls the Pochhammer dispersion equation for slender rods -which is later used to compute the reference dispersion curve- and details the way it is solved. Section 4 then gives the correct dispersion curve for the four-mode equation and examines the convergence of the approximate dispersion curves with an increasing number of degrees of freedom (modes). Lastly, Section 5 contains a discussion on the advantages and drawbacks of using either directly the Pochhammer equation or the Jacobi polynomial approximation, we also discuss the computation cost of both methods.

\section{Dimensionless equation}

\subsection{Assumptions}

We study the longitudinal stress wave propagation in a slender bar of radius $a$. A cylindrical coordinate system $(r, \theta, x)$ is chosen: $r$ denotes the radial distance and $\theta$ is the angular coordinate, $x$ is the axial coordinate along the bar axis (longitudinal axis).

\footnotetext{
${ }^{*}$ Corresponding author

Email addresses: denis.brizard@ifsttar.fr (D. Brizard), eric.jacquelin@univ-lyon1.fr (E. Jacquelin), sylvie.ronel@univ-lyon1.fr (S. Ronel) 
The medium is supposed to be homogeneous, isotropic, and elastic. The elasticity is described either with $(E, \nu)$ (Young modulus and Poisson coefficient) or with $(\lambda, \mu)$, the Lamé constants. As a reminder, the following relationships hold

$$
\lambda=\frac{\nu E}{(1-2 \nu)(1+\nu)} \text { and } \mu=\frac{E}{2(1+\nu)} .
$$

Further, the displacements and the deformations are assumed to be small. The requested displacement field is axisymmetrical: $\mathbf{u}(r, \theta, x, t)=\mathbf{u}(r, x, t)$. The external surface $(r=a)$ is stress free.

\subsection{Governing equations}

The constitutive equations of an elastic medium are [10]

$$
\begin{aligned}
& \sigma_{x x}=\lambda \Delta+2 \mu \epsilon_{x x}, \\
& \sigma_{r r}=\lambda \Delta+2 \mu \epsilon_{r r}, \\
& \sigma_{\theta \theta}=\lambda \Delta+2 \mu \epsilon_{\theta \theta}, \\
& \sigma_{x r}=\mu \epsilon_{x r},
\end{aligned}
$$

where $\Delta=\epsilon_{x x}+\epsilon_{r r}+\epsilon_{\theta \theta}$.

The displacements are assumed to be small, then the deformations are defined as

$$
\begin{aligned}
\epsilon_{x x} & =u_{x, x}, \\
\epsilon_{r r} & =u_{r, r}, \\
\epsilon_{\theta \theta} & =\frac{1}{r}\left(u_{r}+u_{\theta, \theta}\right), \\
\epsilon_{x r} & =u_{r, x}+u_{x, r},
\end{aligned}
$$

where $q_{, \alpha}=\partial q / \partial \alpha$. Further, the circumferential displacement $u_{\theta}$ is zero due to symmetry.

Finally, the equations of motion of an axisymmetrical 3D medium are

$$
\begin{array}{r}
\sigma_{x r, r}+\sigma_{x x, x}+\frac{\sigma_{x r}}{r}=\rho \frac{\partial^{2} u_{x}}{\partial t^{2}}, \\
\sigma_{r r, r}+\sigma_{x r, x}+\frac{\sigma_{r r}-\sigma_{\theta \theta}}{r}=\rho \frac{\partial^{2} u_{r}}{\partial t^{2}} .
\end{array}
$$

The external surface is stress-free, then the boundary conditions are written

$$
\begin{aligned}
& \sigma_{x r}(a, x, t)=0, \forall x, \forall t, \\
& \sigma_{r r}(a, x, t)=0, \forall x, \forall t .
\end{aligned}
$$

Further, we are interested in harmonic plane wave propagation in the $x$-direction, then the solution $\mathbf{u}(x, r, t)$ is such that

$$
\mathbf{u}(x, r, t)=\mathbf{U}(r) \exp (\gamma x+\imath \omega t)
$$

where $\gamma$ is the propagation coefficient whose real part is the attenuation coefficient and imaginary part is the wavenumber $(\gamma=\alpha+\imath k) ; \omega$ is the circular frequency of the propagating wave.

\subsection{Dimensional analysis}

The set of the dimensionless parameters and variables is not unique; it depends on the set of parameter$\mathrm{s} /$ variables that are chosen as the reference parameters. The number of the parameter of reference is given by the number of the fundamental dimensions that occur in the definition of the problem. In dynamics, the fundamental dimensions are usually the length $[L]$, the mass $[M]$, and the time $[T]$.

It is therefore important to list the parameters and variables and to identify their fundamental dimensions. The parameters and variables are listed in Table 1 and $a, E$ and $\rho$ are the three reference parameters. All 


\begin{tabular}{ccc}
\hline parameter/variable & dimension & dimensionless parameter/variable \\
\hline$E$ & {$[M][L]^{-1}[T]^{-2}$} & 1 \\
$a$ & {$[L]$} & 1 \\
$\rho$ & {$[M][L]^{-3}$} & 1 \\
$\nu$ & {$[-]^{-1}[T]^{-2}$} & $\nu$ \\
$\lambda$ & {$[M][L]^{-2}$} & $\tilde{\lambda}=\lambda / E$ \\
$\mu$ & {$[M][L]^{-1}[T]^{-2}$} & $\tilde{\mu}=\mu / E$ \\
$\underline{\bar{\epsilon}}$ & {$[M][L]^{-1}[T]^{-2}$} & $\underline{\tilde{\sigma}}=\underline{\underline{\sigma}} / E$ \\
$\underline{\underline{\epsilon}}$ & {$[-]$} & $\underline{\underline{\epsilon}}$ \\
$r$ & {$[L]$} & $\tilde{x}=r / a$ \\
$\mathbf{u}$ & {$[L]$} & $\tilde{\mathbf{u}}=\mathbf{u} / a$ \\
$c$ & {$[L]$} & $\tilde{c}=c \sqrt{\rho / E}=c / c_{0}$ \\
$t$ & {$[L][T]^{-1}$} & $\tilde{t}=t \sqrt{E / \rho} / a=t c_{0} / a$ \\
$\gamma$ & {$[M]^{-1}$} & $\tilde{\gamma}=\gamma \mathrm{a}$ \\
$\omega$ & {$[T]^{-1}$} & $\tilde{\omega}=\omega a \sqrt{\rho / E}=\omega a / c_{0}$ \\
\hline
\end{tabular}

Table 1: Dimensionless parameters and variables

the other quantities are made dimensionless by combining the quantity with these three reference parameters (see Table 1): the corresponding dimensionless variable to variable $q$ is denoted $\tilde{q}$. To simplify the notations quantity $c_{0}$ is introduced, which has the dimension of a velocity: it corresponds to the velocity of longitudinal waves.

The definition of $c_{0}$ is a consequence of the choice of reference parameters. If, instead of dividing the stresses by the Young Modulus, they were divided by $\mu$ (for example), $c_{0}$ would have another definition and would be the shear wave velocity, $c_{0}=\sqrt{\mu / \rho}$.

\subsection{Dimensionless equations}

From Table 1 it is possible to derive the dimensionless equations. The derivative with respect to the dimensionless coordinates are

$$
\frac{\partial}{\partial \tilde{x}}=a \frac{\partial}{\partial x} \text { and } \frac{\partial}{\partial \tilde{r}}=a \frac{\partial}{\partial r}
$$

Therefore, deformations, as dimensionless quantities, are not affected by the transformations:

$$
\begin{aligned}
\epsilon_{x x} & =u_{x, x}=\tilde{u}_{x, \tilde{x}}, \\
\epsilon_{r r} & =u_{r, r}=\tilde{u}_{r, \tilde{r}}, \\
\epsilon_{\theta \theta} & =\frac{u_{r}}{r}=\frac{\tilde{u}_{r}}{\tilde{r}}, \\
\epsilon_{x r} & =u_{r, x}+u_{x, r}=\tilde{u}_{r, \tilde{x}}+\tilde{u}_{x, \tilde{r}} .
\end{aligned}
$$

The dimensionless constitutive equations are easily derived

$$
\begin{aligned}
\tilde{\sigma}_{x x} & =\tilde{\lambda} \Delta+2 \tilde{\mu} \epsilon_{x x}, \\
\tilde{\sigma}_{r r} & =\tilde{\lambda} \Delta+2 \tilde{\mu} \epsilon_{r r}, \\
\tilde{\sigma}_{\theta \theta} & =\tilde{\lambda} \Delta+2 \tilde{\mu} \epsilon_{\theta \theta}, \\
\tilde{\sigma}_{x r} & =\tilde{\mu} \epsilon_{x r} .
\end{aligned}
$$


Substituting the dimensionless parameters, variables, and coordinates in equations of motion Eqs. (10) 11) gives the dimensionless equations of motion

$$
\begin{array}{r}
\tilde{\sigma}_{x r, \tilde{r}}+\tilde{\sigma}_{x x, \tilde{x}}+\frac{\tilde{\sigma}_{x r}}{\tilde{r}}=\frac{\partial^{2} \tilde{u}_{x}}{\partial \tilde{t}^{2}}, \\
\tilde{\sigma}_{r r, \tilde{r}}+\tilde{\sigma}_{x r, \tilde{x}}+\frac{\tilde{\sigma}_{r r}-\tilde{\sigma}_{\theta \theta}}{\tilde{r}}=\frac{\partial^{2} \tilde{u}_{r}}{\partial \tilde{t}^{2}} .
\end{array}
$$

The dimensionless boundary conditions related to Eqs. (12]13) are

$$
\begin{aligned}
& \tilde{\sigma}_{x r}(1, \tilde{x}, \tilde{t})=0, \forall \tilde{x}, \forall \tilde{t}, \\
& \tilde{\sigma}_{r r}(1, \tilde{x}, \tilde{t})=0, \forall \tilde{x}, \forall \tilde{t} .
\end{aligned}
$$

\subsection{Polynomial approximation}

Following Mindlin et al. [7] and Anderson [8], the displacement field is expanded in function of Jacobi orthogonal polynomials

$$
\begin{aligned}
& u_{x}(r, x, t)=\sum_{n=0}^{+\infty} U_{n}(r / a) u_{n} \exp (\gamma x+\imath \omega t), \\
& u_{r}(r, x, t)=\sum_{n=0}^{+\infty} V_{n}(r / a) v_{n} \exp (\gamma x+\imath \omega t),
\end{aligned}
$$

where the polynomials are defined as

$$
\begin{aligned}
U_{n}(\tilde{r}) & =\sum_{j=0}^{n}\left(\begin{array}{c}
n \\
j
\end{array}\right) \frac{(n+j) !}{n ! j !} \tilde{r}^{2 j}, \\
V_{n}(\tilde{r}) & =\sum_{j=0}^{n}\left(\begin{array}{c}
n \\
j
\end{array}\right) \frac{(n+j+1) !}{(n+1) !(j+1) !} \tilde{r}^{2 j+1} .
\end{aligned}
$$

They are orthogonal with respect to the weight $\tilde{r}$ on the interval $[0,1]$ [8]. Therefore the dimensionless displacements are also expanded in function of the same polynomials family

$$
\begin{aligned}
& \tilde{u}_{x}(\tilde{r}, \tilde{x}, \tilde{t})=\sum_{n=0}^{+\infty} U_{n}(\tilde{r}) \tilde{u}_{n} \exp (\tilde{\gamma} \tilde{x}+\imath \tilde{\omega} \tilde{t}), \\
& \tilde{u}_{r}(\tilde{r}, \tilde{x}, \tilde{t})=\sum_{n=0}^{+\infty} V_{n}(\tilde{r}) \tilde{v}_{n} \exp (\tilde{\gamma} \tilde{x}+\imath \tilde{\omega} \tilde{t}) .
\end{aligned}
$$

Fig. 11illustrates the first axial and radial polynomial displacements.

The orthogonality properties are applied by multiplying Eq. (24) (resp. Eq. (25)) by $\tilde{r} U_{n}(\tilde{r})$ (resp. $\left.\tilde{r} V_{n}(\tilde{r})\right)$ and by integrating over the interval $[0,1]$

$$
\begin{array}{r}
\int_{0}^{1} \tilde{r} U_{n}(\tilde{r})\left(\tilde{\sigma}_{x r, \tilde{r}}+\tilde{\sigma}_{x x, \tilde{x}}+\frac{\tilde{\sigma}_{x r}}{\tilde{r}}\right) \mathrm{d} \tilde{r}=\int_{0}^{1} \tilde{r} U_{n}(\tilde{r}) \frac{\partial^{2} \tilde{u}_{x}}{\partial \tilde{t}^{2}} \mathrm{~d} \tilde{r}, \\
\int_{0}^{1} \tilde{r} V_{n}(\tilde{r})\left(\tilde{\sigma}_{r r, \tilde{r}}+\tilde{\sigma}_{x r, \tilde{x}}+\frac{\tilde{\sigma}_{r r}-\tilde{\sigma}_{\theta \theta}}{\tilde{r}}\right) \mathrm{d} \tilde{r}=\int_{0}^{1} \tilde{r} V_{n}(\tilde{r}) \frac{\partial^{2} \tilde{u}_{r}}{\partial \tilde{t}^{2}} \mathrm{~d} \tilde{r} .
\end{array}
$$

In addition, the boundary conditions must be included in the equations. This is done by integrating by parts some terms of the left hand side of Eqs. (34+35)

$$
\begin{array}{r}
\int_{0}^{1}\left(\tilde{r} U_{n}(\tilde{r}) \tilde{\sigma}_{x x, \tilde{x}}-\frac{\mathrm{d} U_{n}(\tilde{r})}{\mathrm{d} \tilde{r}} \tilde{r} \tilde{\sigma}_{x r}\right) \mathrm{d} \tilde{r}=\int_{0}^{1} \tilde{r} U_{n}(\tilde{r}) \frac{\partial^{2} \tilde{u}_{x}}{\partial \tilde{t}^{2}} \mathrm{~d} \tilde{r}, \\
\int_{0}^{1}\left(\tilde{r} V_{n}(\tilde{r}) \tilde{\sigma}_{x r, \tilde{x}}-V_{n}(\tilde{r}) \tilde{\sigma}_{\theta \theta}-\frac{\mathrm{d} V_{n}(\tilde{r})}{\mathrm{d} \tilde{r}} \tilde{r} \tilde{\sigma}_{r r}\right) \mathrm{d} \tilde{r}=\int_{0}^{1} \tilde{r} V_{n}(\tilde{r}) \frac{\partial^{2} \tilde{u}_{r}}{\partial \tilde{t}^{2}} \mathrm{~d} \tilde{r} .
\end{array}
$$



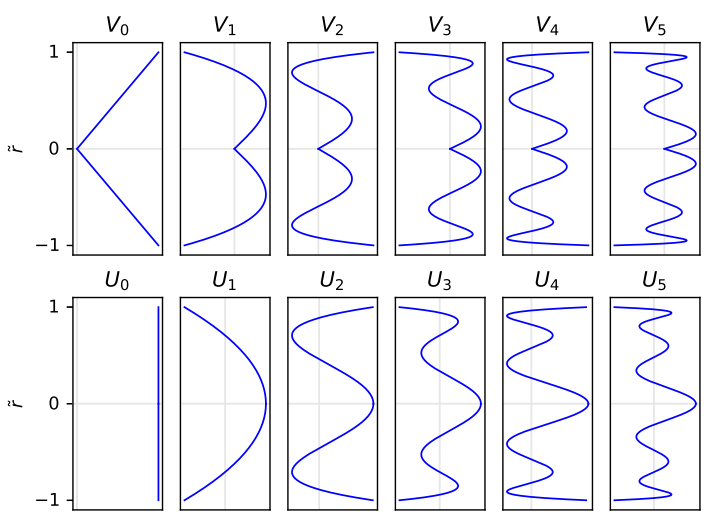

Figure 1: First six radial $\left(V_{n}\right)$ and axial $\left(U_{n}\right)$ deformation modes

Expansions Eqs. (32 33) are truncated up to rank $N$, substituted first in Eqs. (16)23) and then in Eqs. (3637). A homogeneous system of equations with unknowns $\left\{\tilde{u}_{n}, \tilde{v}_{n}\right\}, n=0 \ldots N$ is obtained. A truncation up to rank $N$ leads to $p=2(N+1)$ degrees of freedom, also called $p$-modes approximation in the following. A propagating wave exists only if the determinant of the system is zero, which gives the characteristic equation, that is the relationship between $\tilde{\gamma}$ and $\tilde{\omega}$.

For example, for $N=1$ (and therefore $p=4$ ), the matrix $A$ of the four-mode approximation is

$$
A=\left[\begin{array}{cccc}
\tilde{\omega}^{2}+(\tilde{\lambda}+2 \tilde{\mu}) \tilde{\gamma}^{2} & 2 \tilde{\gamma} \tilde{\lambda} & 0 & -\tilde{\gamma} \tilde{\lambda} \\
2 \tilde{\gamma} \tilde{\lambda} & 4(\tilde{\mu}+\tilde{\lambda})-\frac{1}{2}\left(\tilde{\mu} \tilde{\gamma}^{2}+\tilde{\omega}^{2}\right) & 2 \tilde{\gamma} \tilde{\mu} & -2(\tilde{\mu}+\tilde{\lambda}) \\
0 & 2 \tilde{\gamma} \tilde{\mu} & \frac{1}{3}\left((\tilde{\lambda}+2 \tilde{\mu}) \tilde{\gamma}^{2}+\tilde{\omega}^{2}\right)-8 \tilde{\mu} & \tilde{\gamma} \tilde{\lambda} \\
-\tilde{\gamma} \tilde{\lambda} & -2(\tilde{\mu}+\tilde{\lambda}) & \tilde{\gamma} \tilde{\lambda} & 4 \tilde{\lambda}+7 \tilde{\mu}-\frac{1}{16}\left(\tilde{\mu} \tilde{\gamma}^{2}+\tilde{\omega}^{2}\right)
\end{array}\right]
$$

and the characteristic equation is

$$
\operatorname{det}(A)=0
$$

where $\operatorname{det}(A)=P(\tilde{\gamma})=C_{1} \tilde{\gamma}^{8}+C_{2} \tilde{\gamma}^{6}+C_{3} \tilde{\gamma}^{4}+C_{4} \tilde{\gamma}^{2}+C_{5}$ and

$C_{1}=\tilde{\mu}^{2}(\tilde{\lambda}+2 \tilde{\mu})^{2}$,

$C_{2}=\tilde{\mu}(2 \tilde{\mu}+\tilde{\lambda})\left(2 \tilde{\omega}^{2}(\tilde{\lambda}+3 \tilde{\mu})-24 \tilde{\mu}(10 \tilde{\mu}+11 \tilde{\lambda})\right)$,

$C_{3}=\left(\tilde{\lambda}^{2}+13 \tilde{\mu}^{2}+8 \tilde{\mu} \tilde{\lambda}\right) \tilde{\omega}^{4}-24 \tilde{\omega}^{2} \tilde{\mu}\left(42 \tilde{\mu}^{2}+55 \tilde{\mu} \tilde{\lambda}+14 \tilde{\lambda}^{2}\right)+192 \tilde{\mu}^{2}(9 \tilde{\mu}+4 \tilde{\lambda})(2 \tilde{\mu}+3 \tilde{\lambda})$,

$C_{4}=2(\tilde{\lambda}+3 \tilde{\mu}) \tilde{\omega}^{6}-24 \tilde{\omega}^{4}\left(28 \tilde{\mu}^{2}+26 \tilde{\mu} \tilde{\lambda}+3 \tilde{\lambda}^{2}\right)+192 \tilde{\omega}^{2} \tilde{\mu}\left(47 \tilde{\mu}^{2}+62 \tilde{\mu} \tilde{\lambda}+16 \tilde{\lambda}^{2}\right)-9216 \tilde{\mu}^{2}\left(4 \tilde{\mu}^{2}+8 \tilde{\mu} \tilde{\lambda}+3 \tilde{\lambda}^{2}\right)$

$C_{5}=\tilde{\omega}^{2}\left(\tilde{\omega}^{2}-24 \tilde{\mu}\right)\left(\tilde{\omega}^{2}\left(\tilde{\omega}^{2}-120 \tilde{\mu}-72 \tilde{\lambda}\right)+384(\tilde{\mu}+\tilde{\lambda})(\tilde{\lambda}+2 \tilde{\mu})\right)$.

Matrix $A$ and the coefficients of the characteristic polynomial given for $N=1$ illustrate that the dispersion relation only depends on $\tilde{\lambda}$ and $\tilde{\mu}$, that is on the Poisson's ratio only.

\section{Pochhammer equation for longitudinal wave dispersion in cylindrical rods}

\subsection{Dimensionless Pochhammer equation}

The Pochhammer equation for longitudinal wave dispersion in infinite rods of circular cross section can be found in many references (see [5], Eq. (9); same as [1], Eq. (1); or [2], Eq. (6)). Similarly, a dimensionless 
Pochhammer equation can be given. First define the following two dimensionless parameters

$$
\tilde{\alpha}^{2}=\frac{\tilde{\omega}^{2}}{\tilde{\lambda}+2 \tilde{\mu}}-\tilde{\gamma}^{2} \text { and } \tilde{\beta}^{2}=\frac{\tilde{\omega}^{2}}{\tilde{\mu}}-\tilde{\gamma}^{2} .
$$

The dimensionless Pochhammer equation is then

$$
f(\tilde{\gamma})=2 \tilde{\alpha}\left(\tilde{\beta}^{2}+\tilde{\gamma}^{2}\right) \mathrm{J}_{1}(\tilde{\alpha}) \mathrm{J}_{1}(\tilde{\beta})-\left(\tilde{\beta}^{2}-\tilde{\gamma}^{2}\right)^{2} \mathrm{~J}_{0}(\tilde{\alpha}) \mathrm{J}_{1}(\tilde{\beta})-4 \tilde{\gamma}^{2} \tilde{\alpha} \tilde{\beta} \mathrm{J}_{1}(\tilde{\alpha}) \mathrm{J}_{0}(\tilde{\beta})=0
$$

where $J_{0}$ and $J_{1}$ are the Bessel functions of the first kind of order 0 and 1.

\subsection{Numerical solution}

The determination of the dispersion curve for given bar properties requires to find the roots of the frequency Eq. (46) for the circular frequencies $\omega$ of interest. The numerical solving of Eq. (46) involves Newton's method [11] and therefore requires the analytical derivative $f^{\prime}(\gamma)$, which is just a matter of deriving Bessel's functions (the full analytical expression are given in [12]).

We used a prediction-correction algorithm, starting at $\omega=0$ and then increasing $\omega$ step by step; let $\omega_{i}$ denote the value of $\omega$ at step $i$. The prediction step is an extrapolation of the solution from the solutions computed for the previous values of $\omega$. Three extrapolation methods were tested to predict the value of $\gamma_{i}$ : same value as the previous point $\left(\gamma_{i}=\gamma_{i-1}\right)$; linear extrapolation from the last two previous points $\gamma_{i-1}$ and $\gamma_{i-2}$; and, quadratic extrapolation from the last three previous points $\gamma_{i-1}, \gamma_{i-2}$ and $\gamma_{i-3}$. The correction step uses Newton's method where the starting point is given by the prediction step. The main stopping criterion for the correction step is a cessation of monotonic convergence of $|f(\gamma)|$ towards zero, which in fact indicates oscillations around the solution $\gamma$ with a sign change of the imaginary part of $f$ (real part is null for purely elastic material). The full algorithm starts at $\omega=0$, in that case the solution $c / c_{0}=1$ is trivial. See [12] for the validation of the results given by this algorithm for solving Pochhammer's equation.

\section{Results}

The numerical solution to the Pochhammer equation (see Section 3) is considered as the reference solution for the dispersion curve. We examined in a previous paper the quality of this numerical solution [12].

\subsection{Dispersion curve with the four mode approximation}

For ease of comparison, we use the same example as Anderson [8]: a rod with Poisson's ratio $\nu=0.3$. Note that as we are using the dimensionless equations, only $\nu$ is necessary for the solving of Eq. (39).

Fig. 2 gives the dispersion curve with the four mode approximation with both the erroneous polynomial by Anderson and the correct polynomial given in Eqs. (40) to (44). Fig. 2b is a reproduction of Fig. 4(b) from [8]. The correct set of equations for the four-mode theory -in non-dimensionless form as initially given by Anderson- is provided in Appendix A.

The correction of the error does not seem to change the beginning of the dispersion curve, which remains a very good approximation of the true dispersion curve (see Fig. 2a ). The second part of the dispersion curve -after the inflection point of the curve- is however significantly affected by the correction of the erroneous term and the curve does not converge towards the same horizontal asymptote at high frequency (see Fig. 2b).

\subsection{Higher order approximations}

As is often the case, we plot here the dispersion curves as $\tilde{c}=c / c_{0}$ with respect to the inverse of the dimensionless wavelength $1 / \tilde{\Lambda}=a / \Lambda$, where $\Lambda$ is the wavelength.

Fig. 3 illustrates the evolution of the dispersion curve for $\nu=0.3$ while increasing the number of modes from 2 to 14. Fig. 4 gives the relative error on the dispersion curve $c / c_{0}$, where the numerical solution to Pochhammer equation Eq. (46) is taken as the reference.

Order 2 is displayed here only for completeness, it has no practical interest as it is only tangent to the true dispersion curve at $a / \Lambda=0$ but rapidly moves away. All the orders of polynomial approximation seem 


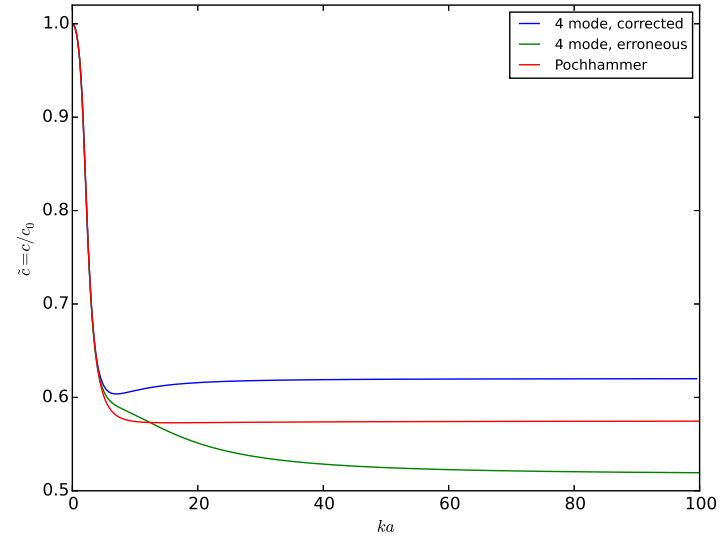

(a)

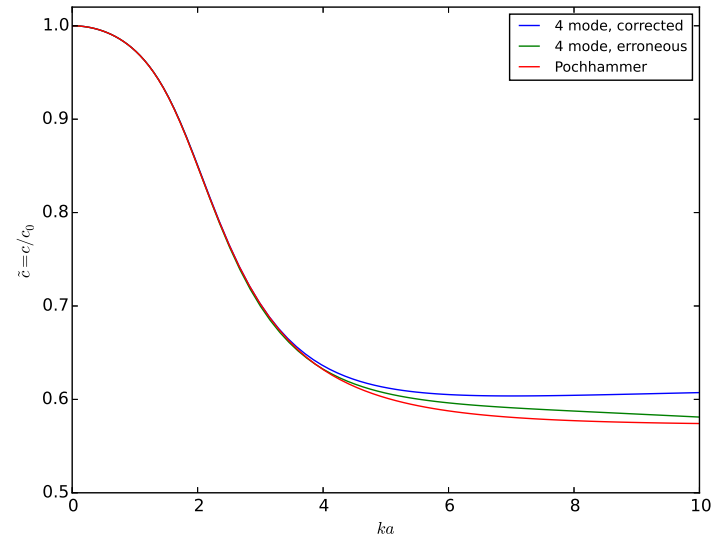

(b)

Figure 2: Dispersion curve with the four mode approximation: (a) full curve; (b) low frequency part

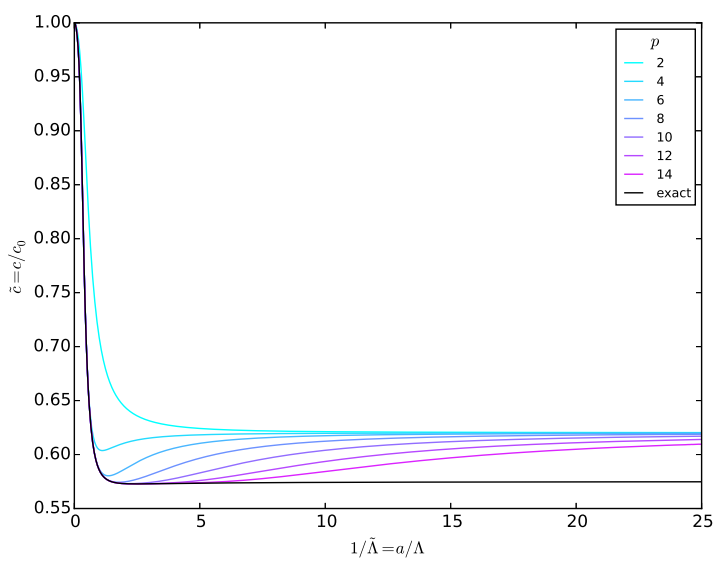

(a)

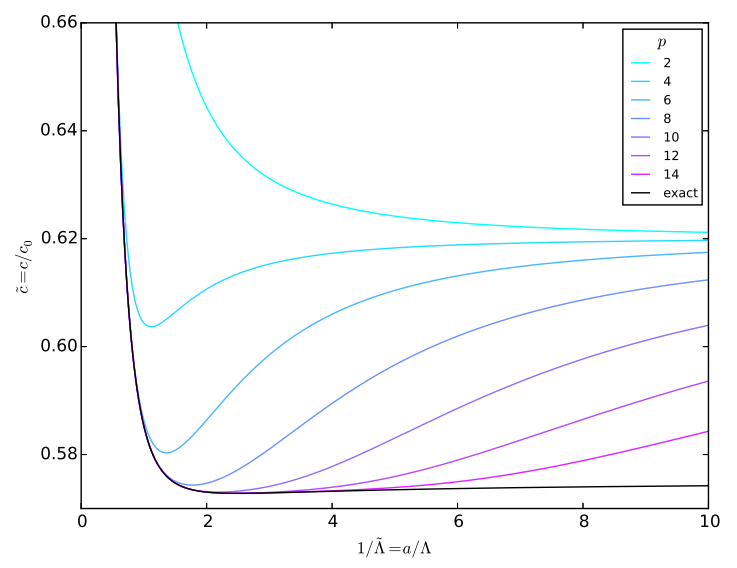

(b)

Figure 3: Dispersion curves with varying order of approximation: (a) full curve; (b) low frequency part 


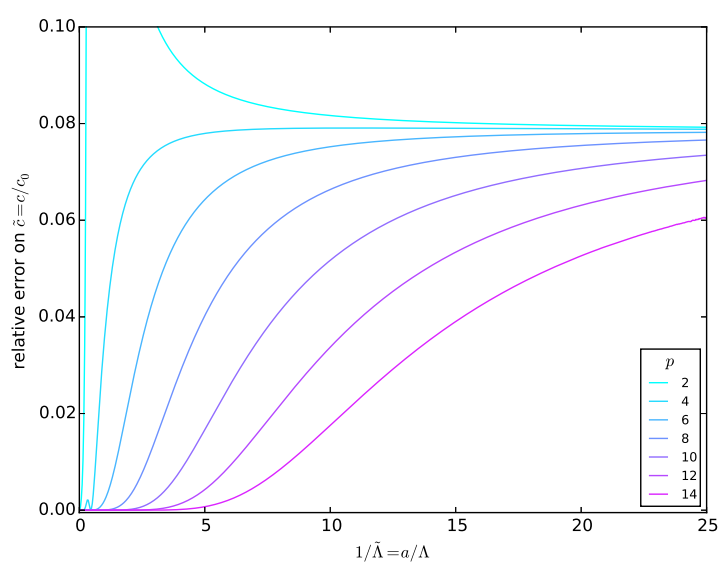

(a)

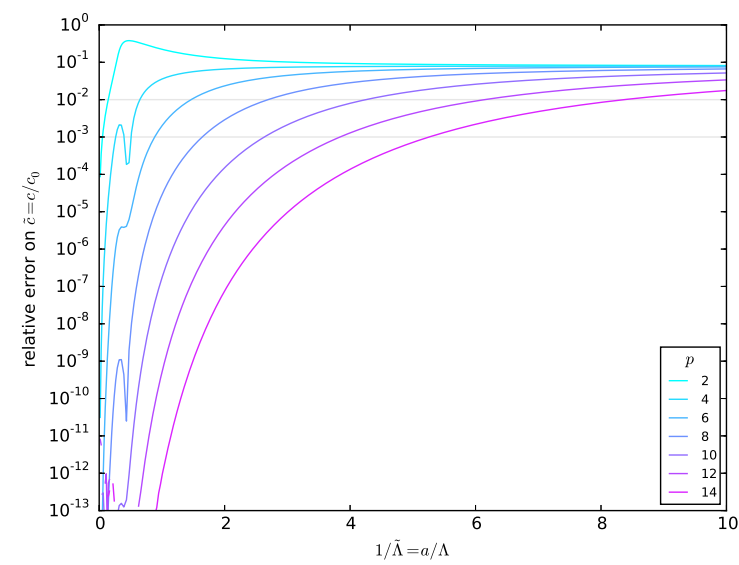

(b)

Figure 4: Relative error on the value of $c / c_{0}$ for different orders of approximation: (a) linear scale; (b) logarithmic scale

\begin{tabular}{rcc}
\hline order & $1 \%$ error & $1 \%$ error \\
\hline 4 & 0.648 & 0.50 \\
6 & 1.46 & 0.88 \\
8 & 2.69 & 1.64 \\
10 & 4.25 & 2.63 \\
12 & 6.14 & 3.84 \\
14 & 8.37 & 5.26 \\
\hline
\end{tabular}

Table 2: Values of $a / \Lambda$ above which the error on $c / c_{0}$ is greater than $1 \%$ and $1 \%$ o

to converge to the same asymptotic value of $c / c_{0}$. Interestingly the dispersion curve for order 2 converges to the same horizontal asymptote as the higher order approximation 1 .

For a given order greater or equal to 4, the relative error on the dispersion curve monotonically increases with the increase of $a / \Lambda$. More precisely, the beginning of the dispersion curve from the polynomial approximation is very close to the Pochhammer dispersion curve, however the error suddenly increases beyond a specific value of $a / \Lambda$. Increasing the order of the polynomial approximation increases the value of $a / \Lambda$ below which the dispersion curve can be considered as satisfactory. Table 2 lists the limit values of $a / \Lambda$ for a maximum relative error on the velocity $c / c_{0}$ of $1 \%$ and $1 \% 0$ and for an approximation of order ranging from 4 to 14 .

\section{Comparison between directly solving Pochhammer equation and polynomial solving}

\subsection{Advantages and drawbacks of solving Pochhammer directly}

The clear advantage of directly solving Pochhammer equation is that one gets the exact solution as no approximation is made. Solving the equation however requires to write a dedicated solving algorithm. We wrote one based on Newton's method but there are other options such as the bisection method. The prediction step may however be critical to get a good convergence of Newton's method towards the required root fo Eq. (46); this point is further discussed in Section 5.3

\footnotetext{
${ }^{1}$ We were not able to derive an analytical expression for this asymptotic value.
} 


\subsection{Advantages and drawbacks of $N$ mode theory}

Using the polynomial approximation makes it very easy to solve the equation as it amounts to find the roots of a polynomial. An approximation is made which leads to an error on the velocity but depending on the maximum frequency of interest, this velocity can be a very good approximation.

Most scientific programming languages have their own function for funding the roots of a polynomial of given degree. For example, Matlab has the roots function and the NumPy Python module contains a Polynomial class which has a roots() method. Most of the root finding algorithms build the companion matrix of the polynomial and then search for its eigenvalues [13].

The only difficulty here is that we are looking for only one of the roots of the polynomial. Depending on the eigenvalue solver behind the function, the roots may not come in the same order when parameter $\omega$ is varying.

Fortunately, the interesting root always has the same index with the Python/Numpy 2 Polynomial class, whatever the value of $\omega$. On the contrary, the old poly1d class from Numpy or the roots function of Matlah 3 do not give the interesting root at the same index.

In that case, it is necessary to follow the interesting root -while $\omega$ increases- by also computing the eigenvectors of matrix $A$ (Equation [38). The counterpart of this eigenvector monitoring is the increase in computational cost. In that case, the polynomial approximation provides a very robust way of computing the dispersion in the bar; indeed we are sure to find the roots of the polynomial approximation. On the contrary, the unconditional convergence of the algorithm for numerically solving the Pochhammer equation is not guaranteed.

\subsection{Computation time}

We compare here the time taken to compute the dispersion curve, either from the Pochhammer equation (see Section 3.2) or from the dimensionless polynomial approximations (see the derivation of the equations in Section 2.5). The computation time are roughly evaluated on an Intel $\AA$ Core ${ }^{\mathrm{TM}} \mathrm{i} 5-3210 \mathrm{M} 2.5 \mathrm{GHz}$ CPU.

Both methods are implemented in Python and make use of the NumPy module. We tried to optimize the code when possible. For the polynomial approximation, the power of $\tilde{\omega}, \tilde{\lambda}$ and $\tilde{\mu}$ is therefore computed only once and than reused (see e.g. Eqs. 40 to 44). The bottleneck however comes from the root finding step. Getting the dispersion curve from the polynomial approximation takes from around $0.15 \mathrm{~s}$ (4 modes) to $0.4 \mathrm{~s}$ (14 modes), for 1000 values of the circular frequency.

Solving the Pochhammer equation takes $0.4 \mathrm{~s}$ for 1000 values of $\omega$, in case of a quadratic extrapolation for the prediction step. The extrapolation method used for the prediction step has a clear effect on the speed of convergence of Newton's method. In case there is not extrapolation (last point), the algorithm fails to follow the dispersion curve and switches from a solution to another (Pochhammer equation has an infinite number of solutions and generally we are interested in the first mode). In case of the linear extrapolation for the prediction step, it takes around $0.50 \mathrm{~s}$ and 3275 Newton iterations to compute 1000 values of $c / c_{0}$ (which means an average of 3.3 iterations per point). The quadratic extrapolation improves the rapidity of convergence of Newton's method by providing a better estimate for the solution: in that case it takes $0.41 \mathrm{~s}$ and 2773 Newton iterations (average 2.8 iterations per point).

\section{Conclusion}

This article gives the right equations for the four-mode theory introduced by Anderson [8]. We also give the associated dispersion curve. The theory was also extended to an arbitrary number of modes 4 and we show the results for a number of modes up to 14 .

The polynomial approximation for the dispersion of longitudinal waves in rod gives very good results in low frequency and increasing the number of modes increases the frequency until which the polynomial

\footnotetext{
${ }^{2}$ This was tested with NumPy version 1.9 .2

${ }^{3}$ This was tested with Matlab version R2018a

${ }^{4}$ The coefficients of the polynomial approximations can be found at https://github.com/dbrizard/polydisp_rod
} 
approximation does not exceed a given level of error. Computing the dispersion curve with the polynomial approximation is more robust than numerically solving the Pochhammer equation. In case there is a need to monitor the solutions given by the polynomial root finding algorithm, the computational cost of monitoring the eigenvectors of matrix $A$ (see Eq. 38) can be a serious drawback.

If computation time matters, we think that using a number of modes greater than 12 is not interesting because solving directly the Pochhammer equation is faster and does not give an approximate solution. However, this also depends on the upper limit for the frequency of interest: if the highest value of $1 / \tilde{\Lambda}$ is 1 , then the six-mode theory is a very good approximation and remains fast to compute. Table 2 may be helpful to make the best compromise between accuracy and computational cost.

This paper is also the occasion to give the equations in dimensionless form: solving the approximate dispersion equation then only relies on Poisson's ratio $\nu$.

\section{Appendix A. Correct coefficients for the four-mode theory}

We give here the right coefficients of the four-mode equation [8]

$$
\begin{gathered}
C_{1}=a^{8} \mu^{2}(2 \mu+\lambda)^{2}, \\
C_{2}=a^{6} \mu(2 \mu+\lambda)\left(2 \rho a^{2} \omega^{2}(3 \mu+\lambda)-24 \mu(10 \mu+11 \lambda)\right), \\
C_{3}=a^{4}\left(\rho^{2} a^{4} \omega^{4}\left(13 \mu^{2}+8 \mu \lambda+\lambda^{2}\right)-24 \rho a^{2} \omega^{2} \mu\left(42 \mu^{2}+55 \mu \lambda+14 \lambda^{2}\right)+192 \mu^{2}(9 \mu+4 \lambda)(2 \mu+3 \lambda)\right), \\
C_{4}=a^{2}\left(2 \rho^{3} a^{6} \omega^{6}(3 \mu+\lambda)-24 \rho^{2} a^{4} \omega^{4}\left(28 \mu^{2}+26 \mu \lambda+3 \lambda^{2}\right)\right. \\
\left.+192 \rho a^{2} \omega^{2} \mu\left(47 \mu^{2}+62 \mu \lambda+16 \lambda^{2}\right)-9216 \mu^{2}\left(4 \mu^{2}+8 \mu \lambda+3 \lambda^{2}\right)\right), \\
C_{5}=\rho a^{2} \omega^{2}\left(\rho a^{2} \omega^{2}-24 \mu\right)\left(\rho a^{2} \omega^{2}\left(\rho a^{2} \omega^{2}-24(5 \mu+3 \lambda)\right)+384(\mu+\lambda)(2 \mu+\lambda)\right) .
\end{gathered}
$$

There is an error in the first line of coefficient $C_{3}$ given by Anderson [8] $(7 \mu \lambda$ instead of $8 \mu \lambda)$, leading to a wrong dispersion curve.

\section{References}

[1] Dennison Bancroft. The Velocity of Longitudinal Waves in Cylindrical Bars. Physical Review, 59(7):588-593, 1941.

[2] Joseph Jr Zemanek. An Experimental and Theoretical Investigation of Elastic Wave Propagation in a Cylinder. The Journal of the Acoustical Society of America, 51(1B):265-283, January 1972.

[3] Bazle A Gama, Sergey L Lopatnikov, and John W Gillespie. Hopkinson bar experimental technique: A critical review. Applied Mechanics Reviews, 57(4):223, 2004.

[4] R. M. Davies. A Critical Study of the Hopkinson Pressure Bar. Philosophical Transactions of the Royal Society of London A: Mathematical, Physical and Engineering Sciences, 240(821):375-457, January 1948.

[5] Han Zhao and Gérard Gary. A three dimensional analytical solution of the longitudinal wave propagation in an infinite linear viscoelastic cylindrical bar. Application to experimental techniques. Journal of the Mechanics and Physics of Solids, 43(8):1335-1348, August 1995.

[6] Augustus Edward Hough Love. A Treatise on the Mathematical theory of Elasticity. New York, Dover Publication, 1944.

[7] R. D. Mindlin and H. D. McNiven. Axially Symmetric Waves in Elastic Rods. Journal of Applied Mechanics, 27(1):145151, March 1960

[8] Simon P. Anderson. Higher-order rod approximations for the propagation of longitudinal stress waves in elastic bars. Journal of Sound and Vibration, 290(1):290-308, February 2006.

[9] John J. Harrigan, Bright Ahonsi, Elisavet Palamidi, and Steve R. Reid. Experimental and numerical investigations on the use of polymer Hopkinson pressure bars. Philosophical Transactions of the Royal Society of London A: Mathematical, Physical and Engineering Sciences, 372(2023):20130201, August 2014.

[10] Karl F. Graff. Wave Motion in Elastic Solids. Dover Publications, June 1991.

[11] Frank W. J. Olver, Daniel W. Lozier, Ronald F. Boisvert, and Charles W. Clark, editors. NIST handbook of mathematical functions. Cambridge University Press, 1 pap/cdr edition, 2010.

[12] D. Brizard and E. Jacquelin. Uncertainty quantification and global sensitivity analysis of longitudinal wave propagation in circular bars. Application to SHPB device. International Journal of Solids and Structures, 134:264-271, March 2018.

[13] James W. Demmel. Applied numerical linear algebra. Society for Industrial and Applied Mathematics, 1 edition, 1997. 